\title{
An Error Bound for Low Order Approximation of Dynamical Systems Subjected to Initial Conditions
}

\author{
G.P.R. MACIEL ${ }^{*}$ and R.S. BARBOSA
}

Received on July 31, 2017 / Accepted on November 21, 2017

\begin{abstract}
In recent years, a great effort has been taken focused on the development of reduced order modeling techniques of dynamical systems. This necessity is pushed by the requirement for efficient numerical techniques for simulations of dynamical systems arising from structural dynamics, controller design, circuit simulation, fluid dynamics and micro electromechanical systems.

We introduce a method to calculate the minimum upper $\mathscr{L}_{2}$ error bound of a linear time invariant reduced order model considering any possible unitary initial conditions (IC). As a consequence, the proposed method calculates the unitary IC vector which leads to the maximum $\mathscr{L}_{2}$ norm of the error. Based on this error bound, it is discussed the capacity of a reduced order system to approximate the free transient response in the worst case scenario.
\end{abstract}

Keywords: Model reduction, dynamical systems, observability grammian, initial condition, error bound.

\section{INTRODUCTION}

Even with the advances in computer technology, which considerably increased computational processing capacity and storage in the last decades, the demand for increasing the complexity of dynamical systems is still a major concern. To handle this problem, a great effort is being taken towards the development of methods to calculate reduced order models (ROM) that reproduce the main dynamic characteristics of a high order model (HOM) with a reduced demand on computational processing capacity, memory and computing time [1, 2, 3, 6, 7, 11, 21].

The determination of error bounds associated with reduced models plays an important role in the development of such Model Order Reduction (MOR) methods. It allows to predict the maximum (or minimum) error associated to the lower order approximation without the need of performing excessively time-demanding simulations. Furthermore, the a priori knowledge of error bounds allows the comparison of different MOR methods and for consequence the selection of the most suitable method to each application based on a prescribed threshold for the ROM error.

*Corresponding author: Gabriel Pedro Ramos Maciel - E-mail: ga.pe.01@gmail.com

Escola politécnica da Universidade de São Paulo, Avenida Professor Mello Moraes, 2231, 05508-970 - São Paulo, SP,

Brazil. E-mail: ga.pe.01@gmail.com; spinola@usp.br 
In an early work, a method is proposed for MOR which guarantees an $\mathscr{L}_{2}$-optimal error bound for the impulse response of Multi-Input-Multi-Output (MIMO) dynamical systems [26].

MOR methods based on modal truncation have advantages compared to other methods due to the physical interpretation of the retained and eliminated eigenmodes, moreover it preserves the original poles of the retained eigenmodes. In the literature one can find proposals of different $\mathscr{H}_{\infty}$-norm error bounds for different modal MOR methods [6, 25, 14].

Another approach to the error bound calculation of ROM is the approximation of the $\mathscr{H}_{2}$ and $\mathscr{H}_{\infty}$ norms for modal MOR methods, found in [12]. This work shows a selection criteria for the eliminated eigenmodes based on the a priori aproximation of the error norms.

A selection criteria was proposed for the truncation of eigenmodes which minimizes the $\mathscr{H}_{2}$ norm of the error associated to modal truncation method [23].

Methods to calculate the $\mathscr{H}_{2}$ and $\mathscr{H}_{\infty}$ error norms for ROM in the balanced realization base are presented in [13] and [1].

A method to compute the suboptimal $\mathscr{H}_{\infty}$-norm error bound shows a suboptimal approach to the task of minimizing the $\mathscr{H}_{\infty}$-norm of the ROM error [19] and have advantages over other Hankel optimal MOR techniques such as better lower error bounds.

A frequency weighted technique for balanced MOR was proposed by Imran et al. [16], which also introduces a frequency weighted $\mathscr{H}_{\infty}$-norm of the ROM error.

In a recent work, an error bound for the MOR of delay linear and nonlinear systems is proposed [24]. This method also preserves the stability in the reduced order model.

The IC problem for nonlinear reduced order dynamical systems was obtained using the Center Manifolds Method [8]. A method is proposed to calculate the initial conditions of the ROM based on de IC of the HOM. Nevertheless, error bounds of the ROM response are not discussed in this work.

Due to the importance of determining error bounds for a ROM, this work introduces a method to calculate the minimum $\mathscr{L}_{2}$-error upper bound for the free transient IC response of a truncated ROM. This upper bound measures the capability of the reduced system to reproduce the free transient IC response of the HOM considering all possible unitary initial conditions, which represents the worst case scenario for this problem. The error bound is first presented regardless the basis onto which the model is projected and than it is showed a specific case for the MOR based on modal coordinates.

\section{PROBLEM STATEMENT}

Consider a linear, time-invariant, asymptotically stable, continuous-time, dynamical system $[\mathbf{A}, \mathbf{B}, \mathbf{C}, \mathbf{D}]$ in its minimal realization, with the following state space representation:

$$
\dot{\mathbf{x}}(t)=\mathbf{A x}(t)+\mathbf{B u}(t) \quad \mathbf{y}(t)=\mathbf{C x}(t)+\mathbf{D u}(t)
$$


Where $\mathbf{x}(t) \in \mathbb{R}^{n}, \mathbf{u}(t) \in \mathbb{R}^{n_{u}}, \mathbf{y}(t) \in \mathbb{R}^{n_{y}}$ are respectively the state vector, input vector and output vector. Hence, $n$ is the order (or state space dimension) of the system and $\mathbf{A} \in \mathbb{R}^{n \times n}, \mathbf{B} \in \mathbb{R}^{n \times n_{u}}$, $\mathbf{C} \in \mathbb{R}^{n_{y} \mathrm{x} n}$ and $\mathbf{D} \in \mathbb{R}^{n_{\mathrm{y}} \mathrm{x} n_{u}}$.

The initial state of the system in eq 2.1 is $\mathbf{x}(0)=\mathbf{x}^{0}$ and the upperscore ${ }^{0}$ in state vectors are herein representing the initial sate (or the IC) of the corresponding system.

This system is herein called a high order dynamical system, or high order model (HOM) of order $n$. It is intended to calculate a $\mathrm{ROM}\left[\mathbf{A}_{r}, \mathbf{B}_{r}, \mathbf{C}_{r}, \mathbf{D}_{r}\right]$ of order $n_{r} \leq n$ which is capable to reproduce the overall dynamic behavior of the HOM.

$$
\dot{\mathbf{x}}_{r}(t)=\mathbf{A}_{r} \mathbf{x}_{r}(t)+\mathbf{B}_{r} \mathbf{u}(t) \quad \mathbf{y}_{r}(t)=\mathbf{C}_{r} \mathbf{x}_{r}(t)+\mathbf{D}_{r} \mathbf{u}(t)
$$

Where $\mathbf{x}_{r} \in \mathbb{R}^{n_{r}}, \mathbf{A}_{\mathbf{r}} \in \mathbb{R}^{n_{r} \times n_{r}}, \mathbf{B} \in \mathbb{R}^{n_{r} \times n_{u}}, \mathbf{C} \in \mathbb{R}^{n_{y} \times n_{r}}$ and $\mathbf{D} \in \mathbb{R}^{n_{y} \times n_{u}}$ and the initial state of this system is $\mathbf{x}_{r}(0)=\mathbf{x}_{r}^{0}$.

Without loss of generality, the HOM can be partitioned onto the most dominant and less dominant Degree of freedom (DOF) $\mathbf{x}_{1} \in \mathbb{R}^{n_{r}}$ and $\mathbf{x}_{2} \in \mathbb{R}^{n-n_{r}}$ respectively:

$$
\left\{\begin{array}{c}
\dot{\mathbf{x}}_{1} \\
\dot{\mathbf{x}}_{2} \\
\hline \mathbf{y}
\end{array}\right\}=\left[\begin{array}{cc|c}
\mathbf{A}_{11} & \mathbf{A}_{12} & \mathbf{B}_{1} \\
\mathbf{A}_{21} & \mathbf{A}_{22} & \mathbf{B}_{2} \\
\hline \mathbf{C}_{1} & \mathbf{C}_{2} & \mathbf{D}
\end{array}\right]\left\{\begin{array}{c}
\mathbf{x}_{1} \\
\mathbf{x}_{2} \\
\hline \mathbf{u}
\end{array}\right\}
$$

It is assumed that the most dominant and less dominant DOF are selected according to an arbitrary selection criterion. A detailed review and descritption of the less dominant DOF selection criteria can be found at $[1,5,18]$.

It is considered that the ROM is obtained by the truncation of the less dominant DOF vector $\mathbf{x}_{2}$. Considering this, $\mathbf{A}_{r}=\mathbf{A}_{11}, \mathbf{B}_{r}=\mathbf{B}_{1}, \mathbf{C}_{r}=\mathbf{C}_{1}$ and $\mathbf{D}_{r}=\mathbf{D}$.

The system which describes the dynamics of the error $\mathbf{e}(t)=\mathbf{y}(t)-\mathbf{y}_{r}(t)$ between the HOM and $\mathrm{ROM}$ is $\left[\mathbf{A}_{e}, \mathbf{B}_{e}, \mathbf{C}_{e}, \mathbf{D}_{e}\right]$, which is:

$$
\left\{\begin{array}{c}
\dot{\mathbf{x}}_{1} \\
\dot{\mathbf{x}}_{2} \\
\dot{\mathbf{x}}_{r} \\
\hline \mathbf{e}
\end{array}\right\}=\left[\begin{array}{ccc|c}
\mathbf{A}_{11} & \mathbf{A}_{12} & \mathbf{0} & \mathbf{B}_{1} \\
\mathbf{A}_{21} & \mathbf{A}_{22} & \mathbf{0} & \mathbf{B}_{2} \\
\mathbf{0} & \mathbf{0} & \mathbf{A}_{11} & \mathbf{B}_{1} \\
\hline \mathbf{C}_{1} & \mathbf{C}_{2} & -\mathbf{C}_{1} & \mathbf{0}
\end{array}\right]\left\{\begin{array}{c}
\mathbf{x}_{1} \\
\mathbf{x}_{2} \\
\mathbf{x}_{r} \\
\hline \mathbf{u}
\end{array}\right\}
$$

Where $\hat{\mathbf{x}}=\left\{\mathbf{x}_{1} ; \mathbf{x}_{2} ; \mathbf{x}_{r}\right\}$ is the state vector for the system in eq. 2.4 and $\mathbf{A}_{e} \in \mathbb{R}^{\left(n+n_{r}\right) \mathrm{x}\left(n+n_{r}\right)}$. The respective IC vector is $\hat{\mathbf{x}}^{0}=\left\{\mathbf{x}_{1}^{0} ; \mathbf{x}_{2}^{0} ; \mathbf{x}_{r}^{0}\right\}$.

The error function is herein defined as the $\mathscr{L}_{2}$ norm of the error vector $\mathbf{e}(t)$, represented by the notation $\|\mathbf{e}\|_{\mathscr{L}_{2}}$. Where

$$
\|\mathbf{e}\|_{\mathscr{L}_{2}}^{2}=\int_{0}^{\infty} \mathbf{e}^{T}(\tau) \mathbf{e}(\tau) d \tau
$$

is the norm in the function space $\mathscr{L}_{2}^{n_{y}}$ of Lebesgue integrable functions $e:[0, \infty) \rightarrow \mathbb{R}^{n_{y}}$. 
Considering this, the intention of the following section is to determine the minimum $\|\mathbf{e}\|_{\mathscr{L}_{2}}$ for the free transient response of the ROM subjected to any possible initial conditions.

$$
\varepsilon_{0}=\max \left\{\|\mathbf{e}\|_{\mathscr{L}_{2}} \quad \mid \quad \hat{\mathbf{x}}^{\mathbf{0}} \in \mathbb{R}^{n+n_{r}} ; \mathbf{u}(t)=0\right\}
$$

\section{A MINIMUM $\mathscr{L}_{2}$-ERROR UPPER BOUND FOR THE IC PROBLEM}

The observability grammian $\mathbf{Q}$ of the system in eq. 2.4 can be calculated using the following Lyapunov equation:

$$
\mathbf{A}_{e}^{T} \mathbf{Q}+\mathbf{Q} \mathbf{A}_{e}+\mathbf{C}_{e}^{T} \mathbf{C}_{e}=0
$$

The observability grammian $\mathbf{Q}$ is symmetric positive semi-definite for asymptotically stable systems and it can be partitioned as

$$
\mathbf{Q}=\left[\begin{array}{lll}
\mathbf{Q}_{11} & \mathbf{Q}_{12} & \mathbf{Q}_{13} \\
\mathbf{Q}_{12}^{T} & \mathbf{Q}_{22} & \mathbf{Q}_{23} \\
\mathbf{Q}_{13}^{T} & \mathbf{Q}_{23}^{T} & \mathbf{Q}_{33}
\end{array}\right]
$$

Where $\mathbf{Q} \in \mathbb{R}^{n+n_{r} \times n+n_{r}}, \mathbf{Q}_{11} \in \mathbb{R}^{n_{r} \times n_{r}}$ and $\mathbf{Q}_{33} \in \mathbb{R}^{n_{r} \times n_{r}}$.

Using the partitioned form in eq. 2.4 and substituting eq. 3.2 in eq. 3.1, the solution for the observability grammian can be decoupled into 3 lower order Lyapunov equations:

$$
\begin{gathered}
\mathbf{A}^{T}\left[\begin{array}{ll}
\mathbf{Q}_{11} & \mathbf{Q}_{12} \\
\mathbf{Q}_{12}^{T} & \mathbf{Q}_{22}
\end{array}\right]+\left[\begin{array}{ll}
\mathbf{Q}_{11} & \mathbf{Q}_{12} \\
\mathbf{Q}_{12}^{T} & \mathbf{Q}_{22}
\end{array}\right] \mathbf{A}+\mathbf{C}^{T} \mathbf{C}=\mathbf{0} \\
\mathbf{A}_{11}^{T}\left[\begin{array}{ll}
\mathbf{Q}_{13}^{T} & \mathbf{Q}_{23}^{T}
\end{array}\right]+\left[\begin{array}{ll}
\mathbf{Q}_{13}^{T} & \mathbf{Q}_{23}^{T}
\end{array}\right] \mathbf{A}-\mathbf{C}_{1}^{T} \mathbf{C}=\mathbf{0} \\
\mathbf{A}_{11}^{T} \mathbf{Q}_{33}+\mathbf{Q}_{33} \mathbf{A}_{11}+\mathbf{C}_{1}^{T} \mathbf{C}_{1}=\mathbf{0}
\end{gathered}
$$

One can observe that the calculated blocks $\left\{\left\{\mathbf{Q}_{11}, \mathbf{Q}_{12}\right\} ;\left\{\mathbf{Q}_{21}, \mathbf{Q}_{22}\right\}\right\}$ in eq. 3.3 corresponds to the observability grammian of the HOM. Analogously, the block $\mathbf{Q}_{33}$ calculated in eq. 3.5 is the observability grammian of the ROM.

Given a non trivial IC to the $\operatorname{HOM} \mathbf{x}^{0} \neq 0$

$$
\mathbf{x}^{0}=\left\{\mathbf{x}_{1}^{0} ; \mathbf{x}_{2}^{0}\right\}
$$

and the IC vector of the system in eq 2.4 is $\hat{\mathbf{x}}^{0}=\left\{\mathbf{x}_{1}^{0} ; \mathbf{x}_{2}^{0} ; \mathbf{x}_{r}^{0}\right\}$.

Considering that the ROM is obtained by the truncation of the least significant DOF of the HOM, the IC vector of the ROM is consequently obtained by the truncation of the IC vector of the HOM:

$$
\mathbf{x}_{r}^{0}=\mathbf{x}_{1}^{0}
$$

From eq. 3.7, the IC vector of the system in eq 2.4 is $\hat{\mathbf{x}}^{0}=\left\{\mathbf{x}_{1}^{0} ; \mathbf{x}_{2}^{0} ; \mathbf{x}_{1}^{0}\right\}$.

It is considered that the error function $\|\mathbf{e}\|_{\mathscr{L}_{2}}$ for the IC problem is:

$$
\|\mathbf{e}\|_{\mathscr{L}_{2}}^{2}=\int_{0}^{\infty} \mathbf{e}^{T}(\tau) \mathbf{e}(\tau) d \tau=\left(\hat{\mathbf{x}}^{0}\right)^{T} \mathbf{Q} \hat{\mathbf{x}}^{0}
$$


Substituting eq. 3.2 into eq. 3.8 and performing simbolic manipulation, the error funcion $\|\mathbf{e}\|_{\mathscr{L}_{2}}$ can be calculated as

$$
\|\mathbf{e}\|_{\mathscr{L}_{2}}^{2}=\left(\mathbf{x}^{0}\right)^{T} \mathbf{Q}_{e} \mathbf{x}^{0}=\left(\mathbf{x}^{0}\right)^{T}\left[\begin{array}{cc}
\mathbf{Q}_{11}+\mathbf{Q}_{13}+\mathbf{Q}_{13}^{T}+\mathbf{Q}_{33} & \mathbf{Q}_{12}+\mathbf{Q}_{23}^{T} \\
\mathbf{Q}_{12}^{T}+\mathbf{Q}_{23} & \mathbf{Q}_{22}
\end{array}\right] \mathbf{x}^{0}
$$

From eq 3.9 , the eq. 2.6 can be rewriten and the minimum upper bound $\varepsilon_{0}$ of $\|\mathbf{e}\|_{\mathscr{L}_{2}}$ for all possible unitary initial conditions is:

$$
\varepsilon_{0}=\max \left\{\|\mathbf{e}\|_{\mathscr{L}_{2}} \quad \mid \quad \mathbf{x}_{0} \in \mathbb{R}^{n} ;\left\|\mathbf{x}_{0}\right\|=1 ; \mathbf{u}(t)=0\right\}
$$

Considering that the solution of eq. 3.1 is positive semidefinite [17] with real nonnegative eigenvalues, the solution of the eq. 3.10 is:

$$
\varepsilon_{0}^{2}=\max _{i} \operatorname{abs}\left(\lambda_{i}\left(\mathbf{Q}_{e}\right)\right)=\operatorname{abs}\left(\lambda_{\max }\left(\mathbf{Q}_{e}\right)\right)
$$

Where $\operatorname{abs}\left(\lambda_{i}\left(\mathbf{Q}_{e}\right)\right)$ is the absolute value of the i-th eigenvalue of $\mathbf{Q}_{e}$ and $\lambda_{\max }\left(\mathbf{Q}_{e}\right)$ is the largest eigenvalue of $\mathbf{Q}_{e}$.

It is important to notice that the eigenvector associated to $\lambda_{\max }\left(\mathbf{Q}_{e}\right)$ is the IC that maximizes the error $\|\mathbf{e}\|_{\mathscr{L}_{2}}$. In other words, it is the scenario which the ROM provides the highest $\mathscr{L}_{2}$-error of the free transient response to the IC problem.

\subsection{Error bound due to initial conditions on modal coordinates}

Modal methods intends to catch the behaviour of the dominant eigenmodes of the HOM into the ROM. In order to do that, the state-space is projected on the subspace spanned by a set of eigenvetors which are considered most dominant [10]. Different selection criteria to elect the most dominant eigenvectors can be found in the literature [21, 25, 12, 15, 22].

Before the truncation of the less dominant states, the HOM can be projected onto an orthonormal modal base [20]. Let us assume that the realization in eq. 2.1 is diagonalizable such that

$$
\dot{\mathbf{w}}(t)=\tilde{\mathbf{A}} \mathbf{w}(t)+\tilde{\mathbf{B}} \mathbf{u}(t) \quad \mathbf{y}(t)=\tilde{\mathbf{C}} \mathbf{w}(t)+\tilde{\mathbf{D}} \mathbf{u}(t)
$$

Where $[\tilde{\mathbf{A}}, \tilde{\mathbf{B}}, \tilde{\mathbf{C}}, \tilde{\mathbf{D}}]$ is the HOM projected onto the modal basis and:

$$
\begin{aligned}
& \mathbf{x}(t)=\Phi \mathbf{w}(t) \\
& \tilde{\mathbf{A}}=\Phi^{-1} \mathbf{A} \Phi=\operatorname{diag}\left(\lambda_{1}, \lambda_{2}, \ldots, \lambda_{n}\right) \\
& \tilde{\mathbf{B}}=\Phi^{-1} \mathbf{B} \\
& \tilde{\mathbf{C}}=\mathbf{C} \Phi \\
& \tilde{\mathbf{D}}=\mathbf{D}
\end{aligned}
$$

Where $\mathbf{w}(t)$ is called modal coordinate vector and $\Phi$ and $\tilde{\mathbf{A}}$ are the eigenvector and eigenvelue matrices respectively. 
Thus, the system in the modal form can be partitioned as in eq. 2.3 with $\mathbf{w}(t)=\left\{\mathbf{w}_{1}(t) ; \mathbf{w}_{2}(t)\right\}$. Where $\mathbf{w}_{1}(t)$ and $\mathbf{w}_{2}(t)$ are the most cominant and less dominant DOF respectively.

$$
\left\{\begin{array}{c}
\dot{\mathbf{w}}_{1} \\
\dot{\mathbf{w}}_{2} \\
\hline \mathbf{y}
\end{array}\right\}=\left[\begin{array}{cc|c}
\tilde{\mathbf{A}}_{11} & \mathbf{0} & \tilde{\mathbf{B}}_{1} \\
\mathbf{0} & \tilde{\mathbf{A}}_{22} & \tilde{\mathbf{B}}_{2} \\
\hline \tilde{\mathbf{C}}_{1} & \tilde{\mathbf{C}}_{2} & \tilde{\mathbf{D}}
\end{array}\right]\left\{\begin{array}{c}
\mathbf{w}_{1} \\
\mathbf{w}_{2} \\
\hline \mathbf{u}
\end{array}\right\}
$$

The reduction by truncation is applied in eq. 3.14 and the error system $\left[\tilde{\mathbf{A}}_{e}, \tilde{\mathbf{B}}_{e}, \tilde{\mathbf{C}}_{e}, \tilde{\mathbf{D}}_{e}\right]$ is

$$
\left\{\begin{array}{c}
\dot{\mathbf{w}}_{1} \\
\dot{\mathbf{w}}_{2} \\
\dot{\mathbf{w}}_{r} \\
\hline \tilde{\mathbf{e}}
\end{array}\right\}=\left[\begin{array}{ccc|c}
\tilde{\mathbf{A}}_{11} & \mathbf{0} & \mathbf{0} & \tilde{\mathbf{B}}_{1} \\
\mathbf{0} & \tilde{\mathbf{A}}_{22} & \mathbf{0} & \tilde{\mathbf{B}}_{2} \\
\mathbf{0} & \mathbf{0} & \tilde{\mathbf{A}}_{11} & \tilde{\mathbf{B}}_{1} \\
\hline \tilde{\mathbf{C}}_{1} & \tilde{\mathbf{C}}_{2} & -\tilde{\mathbf{C}}_{1} & \mathbf{0}
\end{array}\right]\left\{\begin{array}{c}
\mathbf{w}_{1} \\
\mathbf{w}_{2} \\
\mathbf{w}_{r} \\
\hline \mathbf{u}
\end{array}\right\}
$$

With $\hat{\mathbf{w}}=\left\{\mathbf{w}_{1} ; \mathbf{w}_{2} ; \mathbf{w}_{r}\right\}$ is the state vector of eq. 3.15 and $\tilde{\mathbf{e}}(t)$ is the error of the ROM model in the modal basis.

The eq. 3.15 can be simplified to its minimal realization, showed in 3.16.

$$
\left\{\begin{array}{c|c}
\dot{\mathbf{w}}_{2} \\
\hline \tilde{\mathbf{e}}
\end{array}\right\}=\left[\begin{array}{c|c}
\tilde{\mathbf{A}}_{22} & \tilde{\mathbf{B}}_{2} \\
\hline \tilde{\mathbf{C}}_{2} & \mathbf{0}
\end{array}\right]\left\{\begin{array}{c}
\mathbf{w}_{2} \\
\hline \mathbf{u}
\end{array}\right\}
$$

The observability gramian $\tilde{\mathbf{Q}}_{e}$ for the modal form can be calculated from eq. 3.16.

$$
\tilde{\mathbf{A}}_{22}^{T} \tilde{\mathbf{Q}}_{e}+\tilde{\mathbf{Q}}_{e} \tilde{\mathbf{A}}_{22}+\tilde{\mathbf{C}}_{2}^{T} \tilde{\mathbf{C}}_{2}=\mathbf{0}
$$

The method to calculate the observability grammian of the error system using eq. 3.17 requires considerable less computational processing capacity and memory than the method to calculate the grammian using the high order Lyapunov equation to the system in eq. 3.15. The solution of Lyapunov equations involving lower order matrices, as described in this method, is a great advantage considering the high computational effort and memory involved in the solution of this type of problem $[4,9]$

The norm $\|\tilde{\mathbf{e}}\|_{\mathscr{L}_{2}}$ for a given IC $\mathbf{w}_{2}^{0}=\mathbf{w}_{2}(0)$ for eq. 3.16 is

$$
\|\tilde{\mathbf{e}}\|_{\mathscr{L}_{2}}^{2}=\left(\mathbf{w}_{2}^{0}\right)^{T} \tilde{\mathbf{Q}}_{e} \mathbf{w}_{2}^{0}
$$

Consequently, the minimum $\mathscr{L}_{2}$-error upper bound $\tilde{\varepsilon}_{0}$ of the ROM in the modal base, for any given IC $\mathbf{w}(0)$ (applied at the HOM described in eq. 3.15) is

$$
\tilde{\varepsilon}_{0}^{2}=\max _{i} a b s\left(\lambda_{i}\left(\tilde{\mathbf{Q}}_{e}\right)\right)=\operatorname{abs}\left(\lambda_{\max }\left(\tilde{\mathbf{Q}}_{e}\right)\right)
$$

\section{EXAMPLE}

Consider a Single-Input-Single-Output (SISO) dynamical system composed by 3 bodies as in figure 1 . 
The parameter values are: $m_{1}=1 \mathrm{~kg}, m_{2}=0.1 \mathrm{~kg}, m_{3}=1 \mathrm{~kg}, k_{1}=1 \mathrm{~N} / \mathrm{mm}, k_{2}=0.1 \mathrm{~N} / \mathrm{mm}$, $k_{3}=0.5 \mathrm{~N} / \mathrm{mm}, k_{4}=2 \mathrm{~N} / \mathrm{mm}, c_{1}=0.2 \mathrm{~N} /(\mathrm{mm} / \mathrm{s}), c_{2}=0.1 \mathrm{~N} /(\mathrm{mm} / \mathrm{s}), c_{3}=0.1 \mathrm{~N} /(\mathrm{mm} / \mathrm{s}), c_{4}=$ $0.5 \mathrm{~N} /(\mathrm{mm} / \mathrm{s})$.

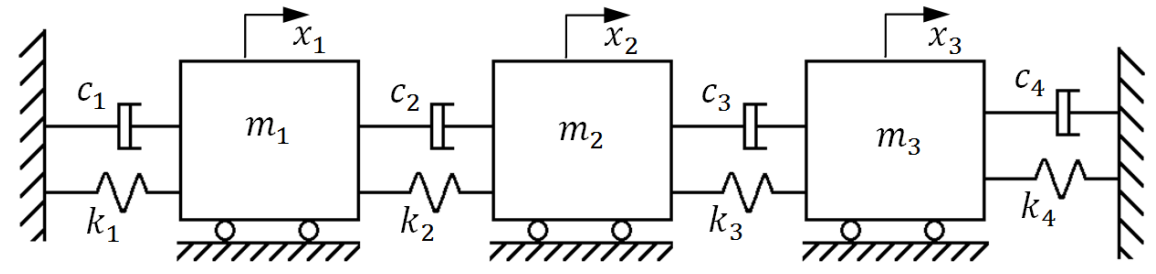

Figure 1: Mass-spring-damper system with 3 bodies.

The state space vetor is $x=\left\{x_{1}, \dot{x}_{1}, x_{2}, \dot{x}_{2}, x_{3}, \dot{x}_{3}\right\}$ and HOM can be written as

$$
\left\{\begin{array}{c}
\dot{x}_{1} \\
\ddot{x}_{1} \\
\dot{x}_{2} \\
\ddot{x}_{2} \\
\dot{x}_{3} \\
\ddot{x}_{3} \\
\hline y
\end{array}\right\}=\left[\begin{array}{cccccc|c}
0 & 1 & 0 & 0 & 0 & 0 & 0 \\
-\frac{k_{1}+k_{2}}{m_{1}} & -\frac{c_{1}+c_{2}}{m_{1}} & \frac{k_{2}}{m_{1}} & \frac{c_{2}}{m_{1}} & 0 & 0 & \frac{1}{m_{1}} \\
0 & 0 & 0 & 1 & 0 & 0 & 0 \\
\frac{k_{2}}{m_{2}} & \frac{c_{2}}{m_{2}} & -\frac{k_{2}+k_{3}}{m_{2}} & -\frac{c_{2}+c_{3}}{m_{2}} & \frac{k_{3}}{m_{2}} & \frac{c_{3}}{m_{2}} & 0 \\
0 & 0 & 0 & 0 & 0 & 1 & 0 \\
0 & 0 & \frac{k_{3}}{m_{3}} & \frac{c_{3}}{m_{3}} & -\frac{k_{3}+k_{4}}{m_{3}} & -\frac{c_{3}+c_{4}}{m_{3}} & 0 \\
\hline 0 & 0 & 1 & 0 & 0 & 0 & 0
\end{array}\right]\left\{\begin{array}{c}
x_{1} \\
\dot{x}_{1} \\
x_{2} \\
\dot{x}_{2} \\
x_{3} \\
\dot{x}_{3} \\
u
\end{array}\right\}
$$

Two reduced models, herein called RO and RM, were calculated by applying the truncation of 2 states in the original and modal basis respectively. Equation 4.2 shows the RO model which state vector is $x_{R O}=\left\{\dot{x}_{R O 1} ; x_{R O 1} ; \dot{x}_{R O 2} ; x_{R O 1}\right\}$

$$
\left\{\begin{array}{c}
\dot{x}_{R O 1} \\
\ddot{x}_{R O 1} \\
\dot{x}_{R O 2} \\
\ddot{x}_{R O 2} \\
\hline y_{R O}
\end{array}\right\}=\left[\begin{array}{cccc|c}
0 & 1.0 & 0 & 0 & 0 \\
-1.1 & -0.3 & 0.1 & 0.1 & 1.0 \\
0 & 0 & 0 & 1.0 & 0 \\
1.0 & 1.0 & -6 & -3 & 0 \\
\hline 0 & 0 & 1.0 & 0 & 0
\end{array}\right]\left\{\begin{array}{c}
x_{R O 1} \\
\dot{x}_{R O 1} \\
x_{R O 2} \\
\dot{x}_{R O 2} \\
\hline u
\end{array}\right\}
$$

Equation 4.3 shows the RM model, obtained by truncating the HOM in the modal basis, which state vector is $w_{R M}=\left\{\dot{w}_{R M 1} ; w_{R M 1} ; \dot{w}_{R M 2} ; w_{R M 1}\right\}$

$$
\left\{\begin{array}{c}
\dot{w}_{R M 1} \\
\ddot{w}_{R M 1} \\
\dot{w}_{R M 2} \\
\ddot{w}_{R M 2} \\
\hline y_{R M}
\end{array}\right\}=\left[\begin{array}{cccc|c}
-0.1248 & 1.0303 & 0 & 0 & 1.4843 \\
-1.0303 & -0.1248 & 0 & 0 & -0.1391 \\
0 & 0 & -0.2962 & 1.3576 & -0.3413 \\
0 & 0 & -1.3576 & -0.2962 & 0.1140 \\
\hline 0.0696 & -0.2477 & -0.0934 & -0.4280 & 0
\end{array}\right]\left\{\begin{array}{c}
w_{R M 1} \\
\dot{w}_{R M 1} \\
w_{R M 2} \\
\dot{w}_{R M 2} \\
\hline u
\end{array}\right\}=
$$




\section{RESULTS}

Although it is not necessary for the determination of the error bounds, complete and reduced models were simulated subjected to IC in order to demonstrate the dynamic behavior of the error in the worst case scenario.

Figure 2 shows the response of the complete and reduced RO models subjected to the IC $\mathbf{x}_{\lambda_{\max }}(0)$ and $\mathbf{x}_{R O \lambda_{\max }}(0)=$ respectively.

$$
\begin{aligned}
& \mathbf{x}_{\lambda_{\max }}(0)=\left\{\begin{array}{llllll}
-0.0136 ; & 0.0895 & -0.0978 & -0.0157 & -0.0832 & -0.4291
\end{array}\right\} \\
& \mathbf{x}_{R O \lambda_{\max }}(0)=\left\{\begin{array}{lllll}
-0.0136 & 0.0895 & -0.0978 & -0.0157
\end{array}\right\}
\end{aligned}
$$

The vector $\mathbf{x}_{\lambda_{\max }}(0)$ is the eigenvector associated to the eigenvalue $\lambda_{\max }\left(\mathbf{Q}_{e}\right)$ specified in eq. 3.11, with $\left\|\mathbf{x}_{\lambda_{\max }}(0)\right\|=1$. Consequently, $\mathbf{x}_{R O \lambda_{\max }}(0)$ is the IC vector for the truncated reduced model.

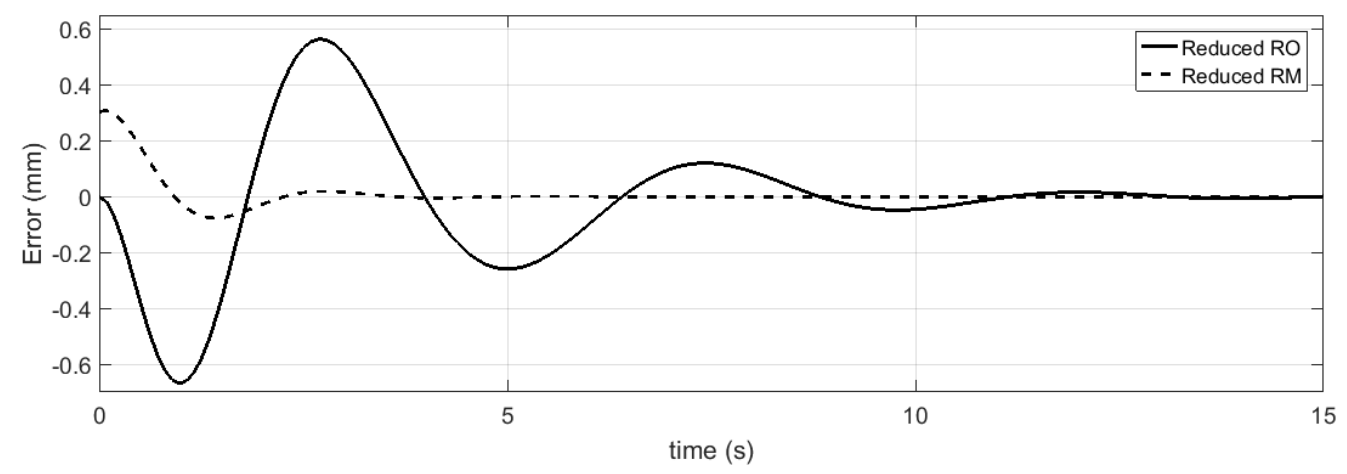

Figure 2: Dynamic simulation of complete and reduced RO system (original basis).

Figure 3 shows the response of the complete and reduced RM models (both in the modal basis) subjected to the IC $\mathbf{w}_{\lambda_{\max }}(0)$ and $\mathbf{w}_{R M \lambda_{\max }}(0)=$ respectively.

$$
\begin{aligned}
& \mathbf{w}_{\lambda_{\max }}(0)=\left\{\begin{array}{llllll}
0 & 0 & 0 & 0 & -0.1611 & 0.9869
\end{array}\right\} \\
& \mathbf{w}_{R M \lambda_{\max }}(0)=\left\{\begin{array}{llll}
0 & 0 & 0 & 0
\end{array}\right\}
\end{aligned}
$$

The vector $\mathbf{w}_{\lambda_{\max }}(0)$ is the eigenvector associated to the eigenvalue $\lambda_{\max }\left(\tilde{\mathbf{Q}}_{e}\right)$ specified in eq. 3.19 , with $\left\|\mathbf{w}_{\lambda_{\max }}(0)\right\|=1$. Consequently, $\mathbf{w}_{R M \lambda_{\max }}(0)$ is the IC vector for the truncated reduced model.

Figure 4 shows the error of the reduced RO and RM systems subjected to the above mentioned initial conditions.

Using eq. 3.11 and eq. 3.19 , the error bounds $\varepsilon_{0}$ and $\tilde{\varepsilon}_{0}$, for the reduced models RO and RM respectively, are presented in Table 1 . These values are compared to the corresponding $\mathscr{L}_{2}$ error norms, calculated from eq. 2.5 . 


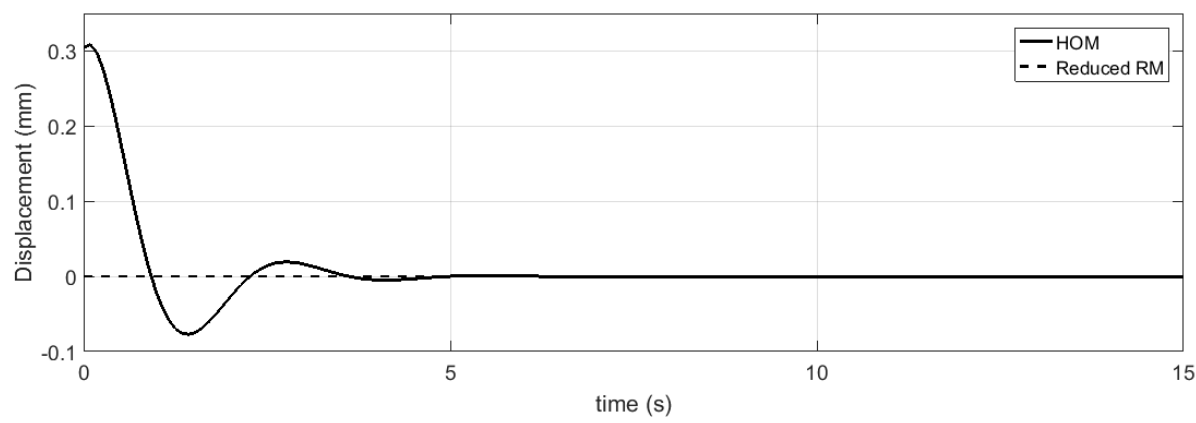

Figure 3: Dynamic simulation of complete and reduced RM system (modal basis).

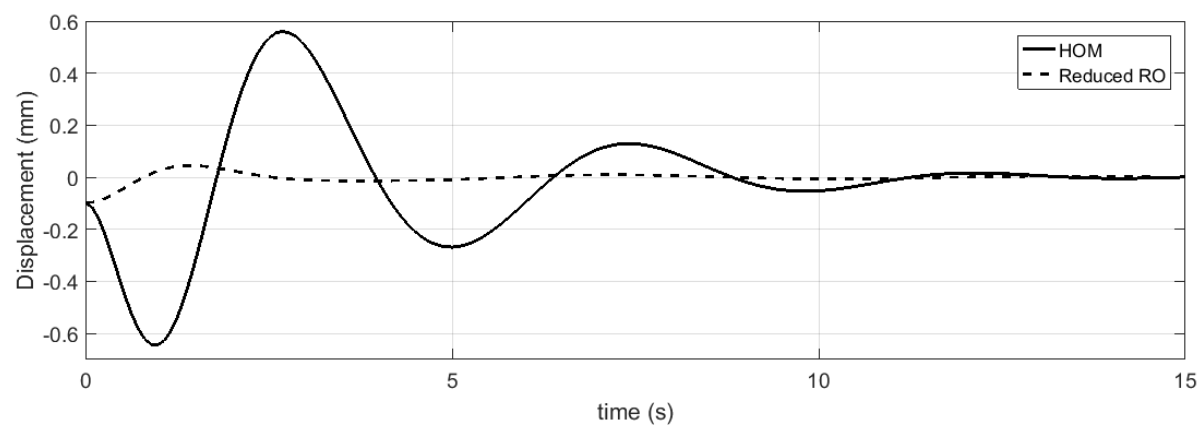

Figure 4: Error of the reduced order systems.

Table 1: Error bounds and $\mathscr{L}_{2}$ error norms calculated from simulations.

\begin{tabular}{ccc}
\hline Reduced model & error bound $^{(1)}$ & $\mathscr{L}_{2}$-norm of the error ${ }^{(2)}$ \\
\hline RO (original basis) & $\varepsilon_{0}=0.8885$ & 0.8885 \\
RM (modal basis) & $\tilde{\varepsilon}_{0}=0.2119$ & 0.2119 \\
\hline
\end{tabular}

(1) calculated using eq. 3.11 and eq. 3.19

(2) calculated using eq. 2.5

\section{CONCLUSIONS}

In this work, a method to calculate an error bound for Model Order Reduction (MOR) by truncation was introduced for the IC problem. This minimum upper bound measures the capability of the Reduced Order Model (ROM) to reproduce the dynamics of the High Order Model (HOM) in the worst scenario. The application was first presented regardless the realization of the HOM. 
Later, the special case for MOR by modal truncation was presented and discussed. The proposed method also have the advantage of a considerable less computational processing capacity and memory to the calculate the observability gramian of the error system. This leads to a considerable time, hardware and memory savings when the method is applied to extremely large systems.

The advantages of using the presented method is that the minimum upper bound of the $\mathscr{L}_{2}$ error can be calculated without the necessity to perform the free transient analysis of the error.

According to the numerical results, the error bound of the reduced model by modal truncation (RM) is smaller than the truncation in the original basis (RO), such a result indicates that the reduction by modal truncation is a better approximation than the truncation in the original basis for the free transient response approximation.

\begin{abstract}
RESUMO. Nos últimos anos, um grande esforço tem sido realizado para se obter modelos de sistemas dinâmicos de ordem reduzida. Esta necessidade é impulsionada pela demanda por técnicas mais eficientes para simular sistemas dinâmicos em áreas como dinâmica de estruturas, projeto de controladores, circuitos eletrônicos, dinâmica de fluidos e sistemas microeletromecânicos.

Os autores propõem um método para calcular o limite superior mínimo da norma $\mathscr{L}_{2}$ do erro de um sistema linear invariante no tempo e de ordem reduzida, considerando todas as possíveis combinações de condições iniciais unitárias. Consequentemente, o método proposto calcula o vetor unitário de condições iniciais que maximiza a norma $\mathscr{L}_{2}$ do erro do sistema reduzido. Baseado neste limite de erro, avalia-se a capacidade que um sistema dinâmico de ordem reduzida possui para aproximar a resposta transitória frente a condições iniciais.
\end{abstract}

Palavras-chave: Redução da ordem de modelos, sistemas dinâmicos, gramiano de observabilidade, condições iniciais, limite de erro.

\title{
REFERENCES
}

[1] A.C. Antoulas. "Approximation of large-scale dynamical systems", volume 6. Siam (2005). doi:10. $1137 / 1.9780898718713$

[2] A.C. Antoulas, D.C. Sorensen \& S. Gugercin. A survey of model reduction methods for large-scale systems. Contemporary mathematics, 280 (2001), 193-220.

[3] Z. Bai. Krylov subspace techniques for reduced-order modeling of large-scale dynamical systems. Applied numerical mathematics, 43(1-2) (2002), 9-44.

[4] U. Baur \& P. Benner. Factorized solution of Lyapunov equations based on hierarchical matrix arithmetic. Computing, 78(3) (2006), 211-234.

[5] U. Baur, P. Benner \& L. Feng. Model Order Reduction for Linear and Nonlinear Systems: A System-Theoretic Perspective. Arch Computat Methods Eng, 21(4) (2014), 331-358. doi:10.1007/ s11831-014-9111-2. 
[6] P. Benner. "Numerical linear algebra for model reduction in control and simulation", volume 29. Wiley Online Library (2006), pp. 275-296.

[7] P. Benner, V. Mehrmann \& D.C. Sorensen. "Dimension reduction of large-scale systems", volume 45. Springer (2005).

[8] S.M. Cox \& A. Roberts. Initial conditions for models of dynamical systems. Physica D: Nonlinear Phenomena, 85(1) (1995), 126-141.

[9] B.N. Datta. "Numerical methods for linear control systems: design and analysis", volume 1. Academic Press (2004).

[10] E. Davison. A method for simplifying linear dynamic systems. IEEE Transactions on automatic control, 11(1) (1966), 93-101.

[11] R.W. Freund. Model reduction methods based on Krylov subspaces. Acta Numerica, 12 (2003), 267319.

[12] W.K. Gawronski. "Dynamics and control of structures: A modal approach". Springer Science \& Business Media (2004).

[13] K. Glover. All optimal Hankel-norm approximations of linear multivariable systems and their $\mathscr{L}_{\infty}$ error bounds. International journal of control, 39(6) (1984), 1115-1193.

[14] M. Green \& D.J. Limebeer. "Linear robust control". Courier Corporation (2012).

[15] C. Gregory. Reduction of large flexible spacecraft models using internal balancing theory. Journal of Guidance, Control, and Dynamics, 7(6) (1984), 725-732.

[16] M. Imran, A. Ghafoor \& V. Sreeram. A frequency weighted model order reduction technique and error bounds. Automatica, 50(12) (2014), 3304-3309. doi:10.1016/j.automatica.2014.10.062.

[17] P. Lancaster \& M. Tismenetsky. "The theory of matrices: with applications". Elsevier (1985).

[18] G.P.R. Maciel. "Métodos de redução de graus de liberdade em sistemas dinâmicos lineares”. Master's thesis, Escola politécnica da Universidade de São Paulo (2015).

[19] A. Megretski. H-infinity model reduction with guaranteed suboptimality bound. In "American Control Conference, 2006”. IEEE (2006), pp. 6-6. doi:10.1109/ACC.2006.1655397.

[20] L. Meirovitch. "Computational methods in structural dynamics", volume 5. Sijthoff \& Noordhoff International Pub (1980).

[21] G. Obinata \& B.D. Anderson. "Model reduction for control system design". Springer Science \& Business Media (2012).

[22] L. Pernebo \& L. Silverman. Model reduction via balanced state space representations. IEEE Transactions on Automatic Control, 27(2) (1982), 382-387.

[23] S. Rahrovani, M.K. Vakilzadeh \& T. Abrahamsson. Modal Dominancy Analysis Based on Modal Contribution to Frequency Response Function $\mathscr{H}_{2}$-Norm. Mechanical Systems and Signal Processing, 48(1) (2014), 218-231. 
[24] N. van de Wouw, W. Michiels \& B. Besselink. Model reduction for delay differential equations with guaranteed stability and error bound. Automatica, 55 (2015), 132-139. doi:10.1016/j.automatica. 2015.02.031.

[25] A. Varga. On modal techniques for model reduction. Technical report, Technical Report TR R13693, Institute of Robotics and System Dynamics, DLR Oberpfaffenhofen, PO Box 1116, D-82230 Wessling, Germany (1993).

[26] D. Wilson. Optimum solution of model-reduction problem. In "Proceedings of the Institution of Electrical Engineers”, volume 117. IET (1970), pp. 1161-1165. doi:10.1049/piee.1970.0227. 